\title{
Structural, elastic, electronic and optical properties of the half-Heusler ScPtSb and YPtSb compounds under pressure|"
}

\author{
M. Radjai ${ }^{1}{ }^{1}$, A. Bouhemadou², D. Maouche ${ }^{2}$ \\ ${ }^{1}$ Laboratory of Physics of Experimental Techniques and Their Applications (LPTEAM), University of Medea, \\ Algeria \\ ${ }^{2}$ Laboratory for Developing New Materials and their Characterization, University of Ferhat Abbas Setif 1 , Setif \\ 19000, Algeria
}

Received June 20, 2021, in final form August 08, 2021

\begin{abstract}
First-principles calculations using the plane-wave pseudopotential method within the generalized gradient approximation method were performed to study the pressure dependence of the structural, elastic, electronic and optical properties for the half-Heusler compounds ScPtSb and YPtSb in a cubic MgAgAs-type structure. The calculations were performed with the inclusion of spin-orbit coupling. The calculated equilibrium lattice parameters are in good agreement with the available experimental and theoretical values. The crystal rigidity and mechanical stability were discussed using the elastic constants and related parameters, namely bulk modulus, shear modulus, Debye temperature, Poisson's coefficient, Young's modulus and isotropic sound velocities. The calculated electronic band structures show that ScPtSb has an indirect gap of $\Gamma-X$ type, whereas YPtSb has a direct band gap of $\Gamma-\Gamma$ type. Furthermore, the effect of pressure on the optical properties, namely the dielectric function, absorption spectrum, refractive index, extinction coefficient, reflectivity and energy-loss spectrum is investigated for both compounds ScPtSb and YPtSb.
\end{abstract}

Key words: ScPtSb, YPtSb, PP-PW method, optical properties, electronic properties, elastic moduli, ab-initio calculations

\section{Introduction}

It is interesting to study ternary half-Heusler compounds due to their specific properties which make them potential candidates for different applications, such as solar cell and thermoelectric applications [1]. Considering their crystal structures, the Heusler alloys are divided into two distinct groups: (i) full Heusler alloys with unit formula $\mathrm{X}_{2} \mathrm{YZ}$ and (ii) the half-Heusler alloys with unit formula $\mathrm{XYZ}$, where $\mathrm{X}$ and $\mathrm{Y}$ atoms are transition metals, while $\mathrm{Z}$ is either a semiconductor or a non-magnetic metal [1]. In recent years, half-Heusler compounds with a narrow band gap and a valence electron number of 18 per unit cell have received a great deal of attention due to their variety and their interesting properties, such as thermoelectrics, thermal, optoelectronics, and spintronics [2-11]. Among the numerous reported studies on the half-Heusler compounds, we mention as examples, the thermodynamic properties of TiNiSn compound [1], the macroscopic and microscopic properties of LuPtSb compound [12], the elastic, mechanical, phonon and thermodynamic properties of $\mathrm{LuAuPb}$ and $\mathrm{YAuPb}$ compound [13], the effect of pressure on the structural, electronic, elastic, vibration and optical properties of $\mathrm{ScXSb}(\mathrm{X}=\mathrm{Ni}, \mathrm{Pd}, \mathrm{Pt})$ compounds [14] and the structural, electronic, thermodynamic and optical properties of XPtSb $(\mathrm{X}=\mathrm{Lu}, \mathrm{Sc})$ compounds [15].

Despite the large number of studies devoted to the exploration of the properties of the half-Heusler, some fundamental properties of the half-Heusler ScPtSb and $\mathrm{YPtSb}$ are insufficiently studied or are not

*Missoum RADJAI: E-mail: mradjai@yahoo.com 
studied at all. The half-Heusler ScPtSb and YPtSb crystallize in the cubic MgAgAs-type structure in the $F-43 m$ (No. 216) space group. ScPtSb and YPtSb compounds obey the 18 valence electron rule, $\mathrm{Sc} / \mathrm{Y}$, $\mathrm{Pt}$ and $\mathrm{Sb}$ atoms are positioned at $4 a, 4 c$ and $4 b$ Wyckoff atomic positions, respectively. J. Oestreich and co-workers recently reported the preparation, crystalline structure and experimental band gap of the half-Heusler compound ScPtSb [16]. Knowledge of the fundamental physical properties of a material, such as the elastic, electronic and optical properties, is required to know its eventual application. Ab initio calculations become a very useful tool to predict the fundamental properties of materials. Thus, is the present work, we explored the structural, elastic, electronic and optical properties of ScPtSb and YPtSb compounds through ab initio calculations in the framework of density functional theory. We have also investigated the pressure effect on the structural, elastic, electronic and optical properties for ScPtSb and YPtSb. The paper is organized as follows. Calculation details are presented in section 2, the theoretical and computational results are described in section 3, and finally, the conclusion is presented in the last section.

\section{Computational details}

All first-principles calculations were conducted using the pseudopotential plane-wave (PP-PW) method based on the density functional theory (DFT), as implemented in Cambridge Serial Total Energy Package (CASTEP) [17]. The exchange-correlation interactions were treated within the GGAPBEsol [18]. The interactions of valence electrons with ion cores were represented by Vanderbilt-type ultra-soft pseudopotentials [19]. The valence-electron configurations for the scandium (yttrium), platinum, and antimony were generated as $\mathrm{Sc}(\mathrm{Y}): 3 s^{2} 3 p^{6} 3 d^{1} 4 s^{2}\left(4 d^{1} 5 s^{2}\right)$, Pt: $5 d^{9} 6 s^{1}$ and $\mathrm{Sb}: 5 s^{2} 5 p^{3}$, respectively. The plane-wave basis set cut-off energy was set as $650 \mathrm{eV}$ and the integration over the Brillion zone was carried out using a $12 \times 12 \times 12$ Monkhorst and Pack [20] k-mesh. The spin-orbit coupling was included. Convergence test demonstrated that these calculation parameters were sufficient to ensure well the convergence of the total energy, the elastic constants and the density of states (DOS). The optimized structural parameters were determined using the Broyden-Fletcher-Goldfarb-Shanno (BFGS) minimization scheme [21] to find the lowest energy structure, with the following convergence thresholds: energy change per atom less than $5 \times 10^{-6} \mathrm{eV} /$ atom, maximum residual force less than $0.01 \mathrm{eV} / \AA$, maximum stress within $0.02 \mathrm{GPa}$ and maximum atom displacement within $5 \times 10^{-4} \AA$.

The single-crystal elastic constants $C_{i j}$ were determined through first-principles calculations of stressstrain data by applying a set of the given homogeneous deformations of finite value and calculating the resulting stress with respect to the optimization of all the internal degrees of freedoms [17]. In order to calculate the optical properties, the Brillouin zone integration was performed using a dense MonkhorstPack grid of $20 \times 20 \times 20$ k-points. The linear optical coefficients, such as the optical reflectivity, extinction coefficient, refractive index, energy-loss spectrum and absorption coefficient, may be computed from the values of $\varepsilon(\omega)$ spectrum.

\section{Results and discussions}

\subsubsection{Structural properties}

The ternary XPtSb (X=Sc, Y) half-Heusler compounds crystalize in the cubic MgAgAs-type structure with space group $F-43 m$ (No. 216) and $Z=4$. The atomic Wyckoff positions are as follows: X: $4 a(0,0,0)$, $\mathrm{Pt}: 4 c(1 / 4,1 / 4,1 / 4)$ and $\mathrm{Sb} 4 b(1 / 2,1 / 2,1 / 2)$. The crystalline structure of the conventional cell of YPtSb is shown in figure 1 .

The first step of the present work was the calculations of the ground state parameters of ScPtSb and YPtSb, including the lattice constant $(a)$ and the bond lengths between the constituent's atoms at $T=0 \mathrm{~K}$ and $P=0 \mathrm{GPa}$, using the available experimental results as starting data and the BFGS technique for relaxing the structural parameters. The bulk module $B$ and its pressure derivative $\left(B^{\prime}\right)$ obtained by fitting the calculated total energy as function of unit cell volume (E-V) to the Birch equation of state EOS [22] and by fitting pressure-volume (P-V) data for different values of the pressure $P$ to the Birch-Murnaghan 


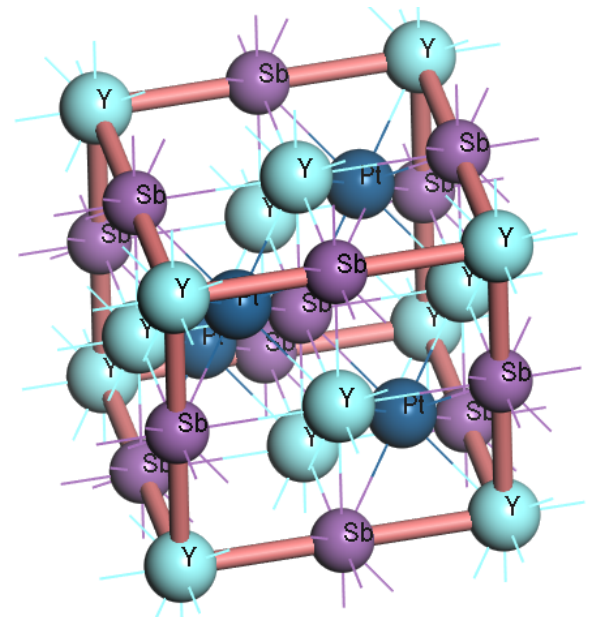

Figure 1. (Colour online) The unit-cell crystalline structure of the YPtSb compound.

$\mathrm{P}-\mathrm{V}$ equation of state EOS [23, 24], Vinet P-V equation of state EOS [25, 26] and Murnaghan P-V equation of state EOS [27], given by the following formulas:

$$
\begin{gathered}
E(V)=E_{0}+\frac{9 B_{0} V_{0}}{16}\left\{\left[\left(V_{0} / V\right)^{2 / 3}-1\right]^{3} B^{\prime}+\left[\left(V_{0} / V\right)^{2 / 3}-1\right]^{2}\left[6-4\left(V_{0} / V\right)^{2 / 3}\right]\right\}, \\
P(V)=\frac{3}{2} B_{0}\left[\left(V_{0} / V\right)^{7 / 3}-\left(V_{0} / V\right)^{5 / 3}\right]\left\{1+\frac{3}{4}\left(B_{0}^{\prime}-4\right)\left[\left(V_{0} / V\right)^{2 / 3}-1\right]\right\}, \\
P(V)=3 B\left(\frac{V_{0}}{V}\right)^{-2 / 3}\left[1-\left(\frac{V_{0}}{V}\right)^{-2 / 3}\right] \exp \left\{-\frac{2}{3}\left(B^{\prime}-1\right)\left[\left(\frac{V_{0}}{V}\right)^{1 / 3}-1\right]\right\}, \\
P(V)=\frac{B}{B^{\prime}}\left[\left(V_{0} / V\right)^{B^{\prime}}-1\right],
\end{gathered}
$$

where $E_{0}$ is the equilibrium energy, $V_{0}$ is the unit-cell volume at zero pressure. The calculated equilibrium structural parameters are listed in table 1 along with the available theoretical and experimental data for the sake of comparison. The E-V and P-V curves are visualized in figure 2 and figure 3 . The calculated lattice parameters for both studied compounds are in very good agreement with the existing experimental and theoretical data. The calculated lattice parameter of ScPtSb (YPtSb) is somewhat smaller than the corresponding experimental value [16] by about $0.03 \%(0.12 \%)$, while it is somewhat larger than previously calculated value [16] by about $0.58 \%(0.13 \%)$. Our calculated bulk modulus for ScPtSb is somewhat larger than the calculated value reported in reference [14], while it is somewhat smaller than that the one reported in [15]. One notes that the calculated bulk modulus of YPtSb is somewhat smaller than that of $\mathrm{ScPtSb}$, which indicates that $\mathrm{YPtSb}$ is more compressible than $\mathrm{ScPtSb}$ and the bulk modulus decreases as the lattice constant increases.

We calculated the lattice parameter $a$ and the cell volume $V$ under a series of pressures in order to evaluate the external hydrostatic pressure effects on the structural properties for both compounds. Variations of $a / a_{0}$ and $V / V_{0}$ as functions of pressure are illustrated in figure 3 , where $a(V)$ is the lattice parameter (unit cell volume) at the considered pressure and $a_{0}\left(V_{0}\right)$ is the corresponding value at zero pressure. The obtained results for $a / a_{0}$ and $V / V_{0}$ in the considered range of pressure are well fitted to a third-order polynomials: 
Table 1. Calculated equilibrium crystal lattice constants $a$ (in $\AA$ ), bulk modulus, $B$ (in GPa), pressure derivative of bulk modulus $B^{\prime}$ (in GPa) and bond length (in $\AA$ ) for ScPtSb and YPtSb. Compared with the available experimental and theoretical data.

\begin{tabular}{|c|c|c|c|c|c|c|}
\hline \multicolumn{7}{|c|}{$\mathrm{ScPtSb}$} \\
\hline Parameter & \multicolumn{2}{|c|}{ Present work } & \multicolumn{2}{|c|}{ Expt } & \multicolumn{2}{|c|}{ Other Cal } \\
\hline$a$ & \multicolumn{2}{|c|}{6.310} & $6.312^{a}$ & $6.310^{b}$ & $6.381^{d}$ & $6.273^{e}$ \\
\hline$B_{0}$ & $\begin{array}{l}124.42^{1} \\
124.43^{3}\end{array}$ & $\begin{array}{l}124.57^{2} \\
124.16^{4}\end{array}$ & \multicolumn{2}{|c|}{ - } & $115.137^{d}$ & $133.465^{e}$ \\
\hline$B_{0}^{\prime}$ & $\begin{array}{l}4.67^{1} \\
4.72^{3}\end{array}$ & $\begin{array}{l}4.76^{2} \\
4.63^{4}\end{array}$ & \multicolumn{2}{|c|}{ - } & $4.82^{d}$ & $5.267^{e}$ \\
\hline $\mathrm{Sb}-\mathrm{Pt}$ & \multicolumn{2}{|c|}{2.732} & & & \multicolumn{2}{|c|}{$2.733^{b}$} \\
\hline $\mathrm{Sb}-\mathrm{Sc}$ & \multicolumn{2}{|c|}{3.155} & & & \multicolumn{2}{|c|}{$3.156^{b}$} \\
\hline Sc-Pt & \multicolumn{2}{|c|}{2.732} & & & \multicolumn{2}{|c|}{$2.733^{b}$} \\
\hline \multicolumn{7}{|c|}{ YPtSb } \\
\hline$a$ & \multicolumn{2}{|c|}{6.524} & $6.538^{c}$ & $6.532^{a}$ & $6.626^{f}$ & $6.533^{g}$ \\
\hline$B_{0}$ & $\begin{array}{l}109.73^{1} \\
109.70^{3}\end{array}$ & $\begin{array}{l}110.03^{2} \\
108.97^{4}\end{array}$ & \multicolumn{2}{|c|}{ - } & \multicolumn{2}{|c|}{ - } \\
\hline$B_{0}^{\prime}$ & $\begin{array}{l}4.72^{1} \\
4.68^{3}\end{array}$ & $\begin{array}{l}4.69^{2} \\
4.67^{4}\end{array}$ & \multicolumn{2}{|c|}{ - } & \multicolumn{2}{|c|}{ - } \\
\hline $\mathrm{Sb}-\mathrm{Pt}$ & \multicolumn{2}{|c|}{2.825} & \multicolumn{2}{|c|}{$2.831^{h}$} & \multicolumn{2}{|c|}{ - } \\
\hline Sb-Y & \multicolumn{2}{|c|}{3.262} & \multicolumn{2}{|c|}{$3.269^{h}$} & \multicolumn{2}{|c|}{ - } \\
\hline Y-Pt & \multicolumn{2}{|c|}{2.825} & & & \multicolumn{2}{|c|}{ - } \\
\hline & 16], ${ }^{b} \operatorname{Ref}$ & $\begin{array}{l}{ }^{1} \text { Presen } \\
{ }^{2} \text { Presen } \\
\text { sent work } \\
\text { Present w } \\
\text { ], }{ }^{c} \mathrm{f}[29] \text {, }\end{array}$ & $\begin{array}{l}\text { rom Bir } \\
\text { rom Vin } \\
\text { irch-Mu } \\
\text { n Murna } \\
4 \text { 4, }{ }^{e} \text { Ref }\end{array}$ & $\begin{array}{l}-\mathrm{V} \text { EOS } \\
-\mathrm{V} \text { EOS } \\
\text { shan P-V } \\
\text { n P-V EC } \\
\mid{ }^{f} \text { Ref | }\end{array}$ & $\begin{array}{l}\text { I, } \\
\mathrm{f}[31],{ }^{h} \mathrm{R}\end{array}$ & 32]. \\
\hline
\end{tabular}
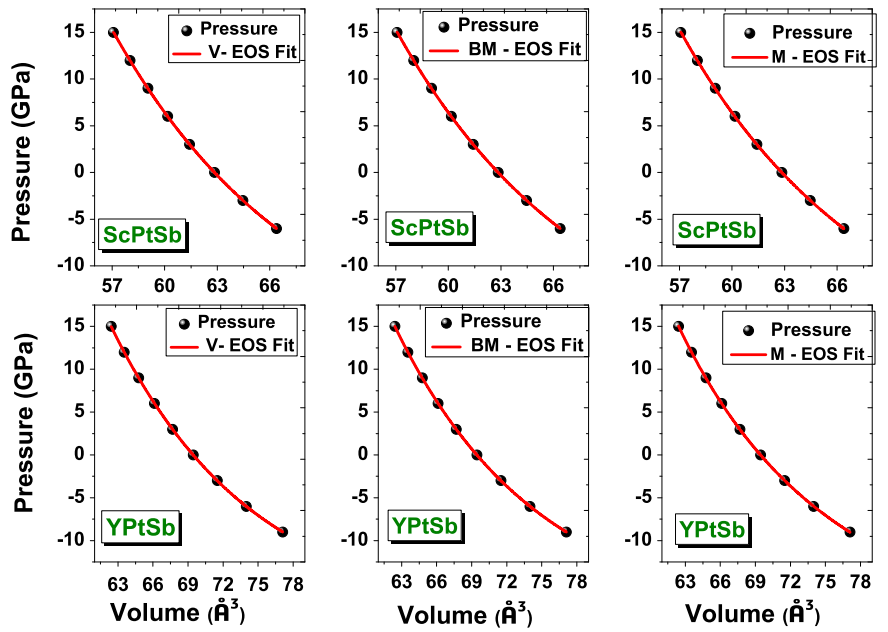

Figure 2. (Colour online) Calculated pressure vs. volume $P(V)$ for the cubic compouds ScPtSb and YPtSb. The solid lines are the fits of the obtained data to the Birch-Murnaghan, Murnaghan and Vinet equations of states. 

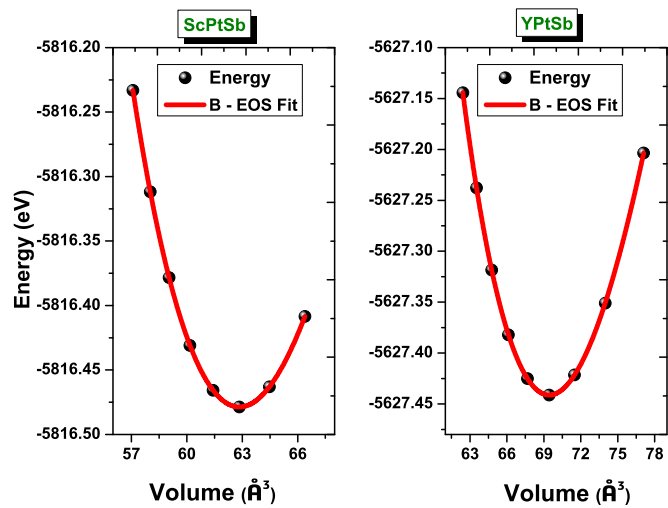

Figure 3. (Colour online) Calculated total energy vs. volume $E(V)$ for the cubic compouds $\mathrm{ScPtSb}$ and YPtSb. The solid lines are the fits of the obtained data to the Bircht equations of states.

$$
\begin{aligned}
& \left\{\begin{array}{l}
a / a_{0}=1-2.65 \times 10^{-3} P+4.67 \times 10^{-5} P^{2}-6.73 \times 10^{-7} P^{3} \\
V / V_{0}=1-7.96 \times 10^{-3} P+1.60 \times 10^{-4} P^{2}-2.46 \times 10^{-6} P^{3},
\end{array}\right. \\
& \left\{\begin{array}{l}
a / a_{0}=1-3.02 \times 10^{-3} P+6.06 \times 10^{-5} P^{2}-9.67 \times 10^{-7} P^{3} \\
V / V_{0}=1-9.04 \times 10^{-3} P+2.04 \times 10^{-4} P^{2}-3.35 \times 10^{-6} P^{3}
\end{array}\right.
\end{aligned}
$$

The obtained volume compressibility $\beta_{V}=7.96 \times 10^{-3} \mathrm{GPa}^{-1}\left(9.04 \times 10^{-3} \mathrm{GPa}^{-1}\right)$ and the linear compressibilities $\beta_{a}=2.65 \times 10^{-3} \mathrm{GPa}^{-1}\left(3.02 \times 10^{-3} \mathrm{GPa}^{-1}\right)$ of the lattice parameters $a$, of the both compounds $\mathrm{ScPtSb}$ (YPtSb) respectively, were used to estimate the bulk modulus $B$ as follows:

$$
\left\{\begin{array}{c}
B_{V}=1 / \beta_{V} \\
B_{a}=1 / 3 \beta_{a}
\end{array}\right.
$$

The values of the bulk modulus $B$ are $B_{V}=125.62 \mathrm{GPa}(110.61 \mathrm{GPa})$ and $B_{a}=125.78 \mathrm{GPa}(110.37 \mathrm{GPa})$ of the $\mathrm{ScPtSb}$ (YPtSb) compounds. These results are in agreement with the bulk module $B$ value extracted from the equation of state (EOS) fit shown in table 1 .
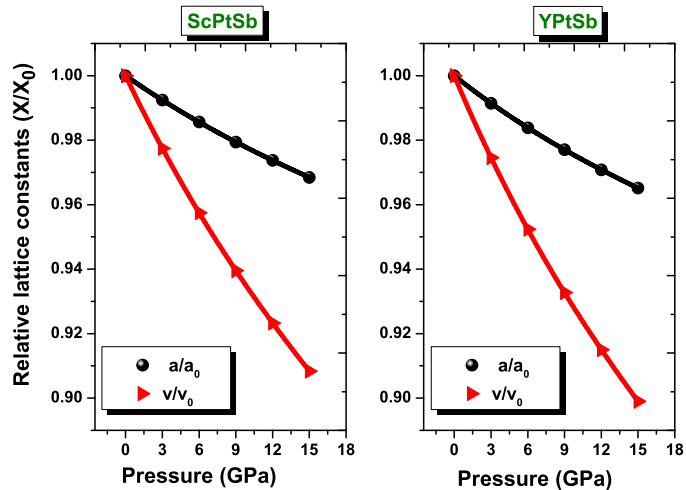

Figure 4. (Colour online) Pressure dependence of the relative variations of the lattice constant $a$ and unit cell volume, $V$, for the $\mathrm{ScPtSb}$ and YPtSb materials. 


\subsection{Elastic properties}

\subsubsection{Single-crystal elastic constants}

The elastic properties can be determined from the elastic constants $C_{i j}$. The $C_{i j}$ characterizes the response of a material to an applied stress [33] and provides information concerning the nature of the forces operating in solids [34]. The elastic behaviour of any cubic crystal is characterized by three independent elastic constants namely, $C_{11}, C_{12}$ and $C_{44}$. The calculated elastic constants of ScPtSb (YPtSb) under zero pressure and zero temperature are $C_{11}=188.62(178.76) \mathrm{GPa}, C_{44}=69.05(54.21) \mathrm{GPa}$ and $C_{12}=92.14(75.26) \mathrm{GPa}$. A cubic crystal is mechanically stable if its elastic constants satisfy the Born criteria: $C_{11}-C_{12}>0, C_{11}>0, C_{44}>0, C_{11}+2 C_{12}>0$. The calculated elastic constants of both studied compounds satisfy the above-mentioned criteria, indicating that these compounds are mechanically stable. In order to understand the mechanical stability, phase transition mechanisms, and interatomic interactions, we studied the effect of pressure on the elastic constants, figure 5 shows the pressure dependence of the three independent elastic constants of the cubic compounds $\mathrm{ScPtSb}$ and $\mathrm{YPtSb}$ for pressures up to $15 \mathrm{GPa}$. The mechanical stability criteria under pressure is expressed as follows [35] $C_{11}-P>\left|C_{12}+P\right|, C_{11}-P>0, C_{44}-P>0, C_{11}+2 C_{12}+P>0$.
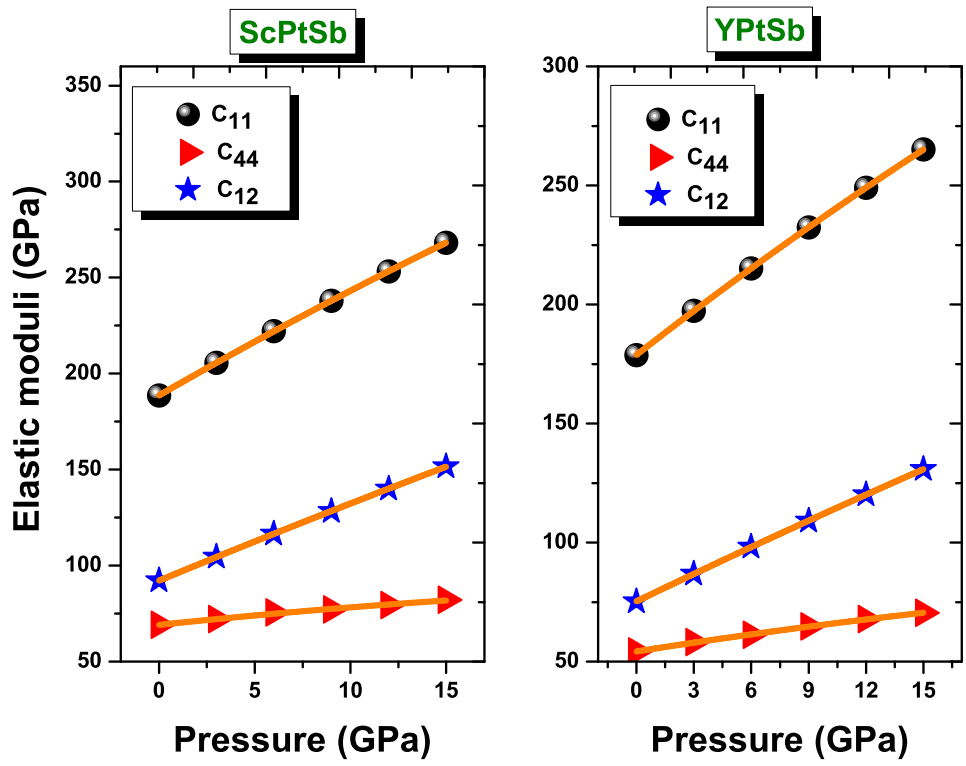

Figure 5. (Colour online) Calculated pressure dependence of the independent elastic constants $C_{i j s}$ for the ScPtSb and YPtSb materials. The symbols indicate the calculated results. The lines represent the results of fitting these theoretical results to a second-order polynomial.

From the obtained results we can make the following conclusions for both investigated compounds: (i) the values of the elastic constants show that $\mathrm{ScPtSb}$ is more resistant to external stress than YPtSb. (ii) The $C_{11}$, which reflects the resistance to unidirectional compression along the a-axis, is much larger than $C_{44}$, which reflects the resistance to shear deformation, indicating that the studied compounds are more resistive to unidirectional compression than to shear deformation. (iii) Mechanical stability restrictions are satisfied for both studied compounds in the pressure range $0-15 \mathrm{GPa}$. (iv) Figure 5 shows that $C_{11}$ is more sensitive to pressure than $C_{12}$ and $C_{44}$. The elastic constants $C_{11}$ and $C_{12}$ increase faster with an increasing pressure than $C_{44}$. Variations of the elastic constants of both studied compounds with 
pressure were fitted with the following polynomials of second order for ScPtSb and YPtSb, respectively:

$$
\left\{\begin{array} { l } 
{ C _ { 1 1 } = 1 8 8 . 6 2 + 5 . 7 3 P - 2 . 9 2 \times 1 0 ^ { - 2 } P ^ { 2 } , } \\
{ C _ { 4 4 } = 6 9 . 0 5 + 1 . 0 4 P - 1 . 3 \times 1 0 ^ { - 2 } P ^ { 2 } , } \\
{ C _ { 1 2 } = 9 2 . 1 4 + 4 . 1 3 P - 1 . 1 7 \times 1 0 ^ { - 2 } P ^ { 2 } , }
\end{array} \quad \left\{\begin{array}{l}
C_{11}=178.76+6.25 P-3.37 \times 10^{-2} P^{2}, \\
C_{44}=54.21+1.26 P-1.22 \times 10^{-2} P^{2}, \\
C_{12}=75.26+3.86 P-1.11 \times 10^{-2} P^{2} .
\end{array}\right.\right.
$$

The isotropic elastic moduli of the polycrystalline phase of a material, such as the bulk $B$, shear moduli $G$, Young's modulus $E$ and Poisson's ratio $v$, can be calculated from its monocrystalline elastic constants $C_{i j}$ via the well-known Voigt-Reuss-Hill (VRH) approximations [36, 37]. Voight and Reuss approximations give upper $\left(B_{V}\right.$ and $\left.G_{V}\right)$ and lower $\left(B_{R}\right.$ and $\left.G_{R}\right)$ limits of the $B$ and $G$ moduli, respectively. For a cubic system, the calculation formulas are as follows [38]:

$$
\left\{\begin{array}{l}
B=\frac{1}{3}\left(C_{11}+2 C_{12}\right), \\
G_{V}=\frac{1}{5}\left(C_{11}-C_{12}+3 C_{15}\right), \\
G_{R}=\left[5\left(C_{11}-C_{12}\right), C_{44}\right] /\left[3\left(C_{11}-C_{12}\right)+4 C_{44}\right], \\
G=\left(G_{V}+G_{R}\right) / 2, \\
E=9 B G /(3 B+G), \\
v=(3 B-E) / 6 B .
\end{array}\right.
$$

The calculated bulk modulus $B$, shear modulus $G$, Young's modulus $E$ and Poisson's ratio $v$ at zero pressure are quoted in table 2. Variations of the polycrystalline bulk modulus $B$, shear modulus $G$, Young's modulus $E, B / G$ ratio and Poisson's ratio $v$ as function of pressure are depicted in figures 6 and 7

Table 2. Calculated bulk modulus $\left(B_{H}\right.$, in $\left.\mathrm{GPa}\right)$; shear modulus $\left(G_{H}\right.$, in GPa); Young's modulus $(E$, in $\mathrm{GPa})$; Poisson's ratio $(v)$ and Debye temperature $\left(\theta_{\mathrm{D}}\right.$, in $\left.\mathrm{K}\right)$ for the $\mathrm{ScPtSb}$ and YPtSb materials.

\begin{tabular}{|c|c|c|c|c|c|c|c|}
\hline System & $B$ & $G_{V}$ & $G_{R}$ & $G$ & $B / G$ & $E$ & $v$ \\
\hline \hline ScPtSb & 124.30 & 60.72 & 58.88 & 59.80 & 2.07 & 154.62 & 0.292 \\
\hline YPtSb & 109.76 & 53.23 & 53.20 & 53.21 & 2.06 & 137.43 & 0.291 \\
\hline
\end{tabular}

The obtained results allow us to make the following conclusions: (i) the values of bulk modulus $B$, shear modulus $G$, Young's modulus $E, B / G$ ratio and Poisson's ratio $v$ increase with an increasing pressure, indicating that their hardness can be improved at a high external pressure. (ii) For both studied compounds, the bulk modulus $B$ is greater than the shear modulus for all the considered pressure range $(0-15 \mathrm{GPa})$. Knowing that the bulk modulus $B$ and shear modulus $G$ mirror the resistance of a material to the volume and shape changes, respectively [39], this result indicates that $\mathrm{ScPtSb}$ and $\mathrm{YPtSb}$ compounds are more resistant to the volume change than to the shape change. (iii) Figure 3 shows that the Young's modulus $E$ (which characterizes the stiffness of solids) of ScPtSb is larger than that of YPtSb, indicating that $\mathrm{ScPtSb}$ is much stiffer than YPtSb. Moreover, the relatively high values of Young's moduli of both compounds indicate the relatively high resistance to uniaxial deformation (compression/ traction). (iv) The values of the bulk modulus for ScPtSb and YPtSb compounds deduced from the single-crystal elastic constants $C_{i j}$ are in good agreement with those calculated from the third order polynomial $P(V)$, and Birch-Murnaghan $P(V)$ EOS, Vinet $P(V)$ EOS, Murnaghan $P(V)$, and Birch $E(V)$ EOS (see table 1 ). This is a strong indication of the reliability of the calculated elastic constants of ScPtSb and YPtSb and the used computational methodology. (v) In order to estimate the brittle or ductile behavior of materials, Pugh [39] introduced the criterion of the ratio of the bulk modulus $B$ to shear modulus $G$. According to this criterion, if $B / G$ value is less than 1.75 brittle, otherwise the material behaves in a ductile manner. The calculated $B / G$ values are all higher than 1.75 for pressures up to $15 \mathrm{GPa}$ as shown in figure 7 , which suggests that both considered materials are of ductile nature. The $B / G$ values of ScPtSb and YPtSb increase with an increasing external pressure, which suggests that the ductility of these compounds increases with an increasing pressure. (vi) Poisson's ratio $v$ is generally connected with the volume 

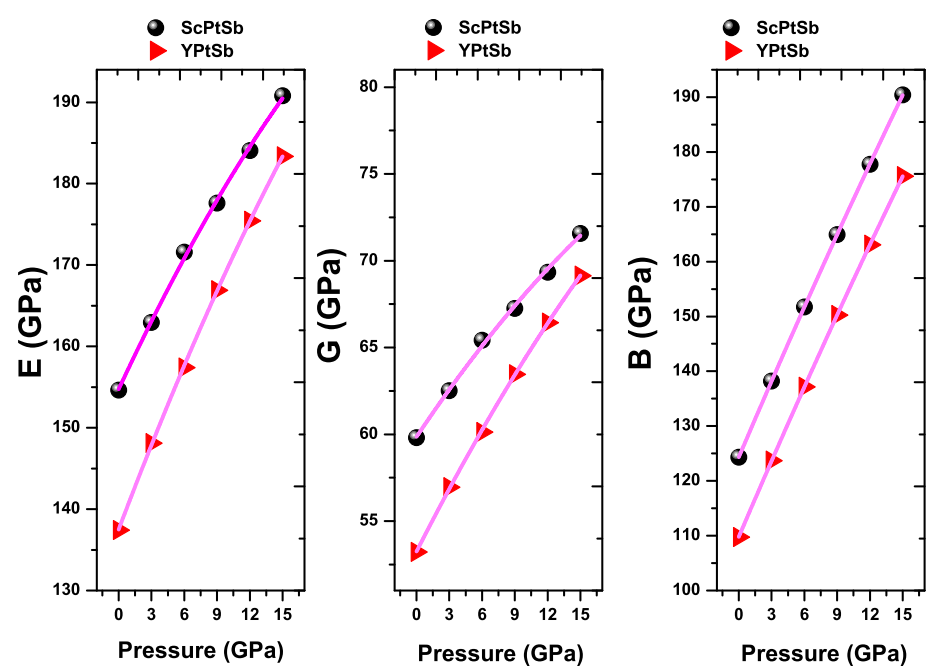

Figure 6. (Colour online) The values of bulk modulus $B$, shear modulus $G$ and Young's modulus $E$ under various pressure.
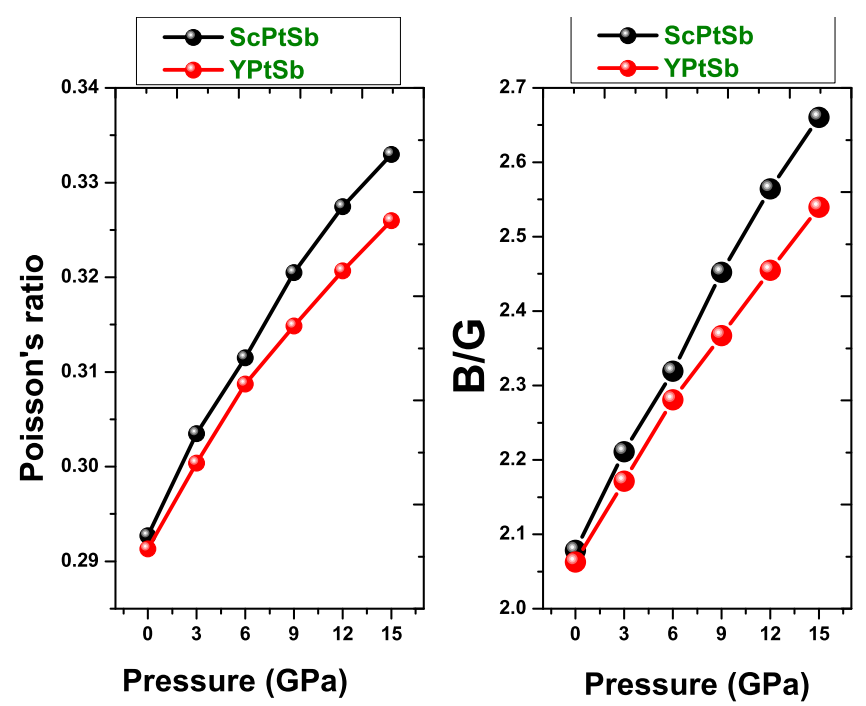

Figure 7. (Colour online) Calculated pressure dependence of Pugh's ratio $B / G$ and Poisson's ratio $v$ for the cubic compounds ScPtSb and YPtSb.

change in a solid during uniaxial deformation [40]. Moreover, $v$ can provide more information about the characteristics of the bonding forces [41], which usually range from 0.25 to 0.5 . The calculated values of $v$ in the considered pressure range $(0-15 \mathrm{GPa})$ are in the range from 0.26 to 0.36 , implying that the interatomic forces are central forces and a considerable volume change can be associated with elastic deformation.

In order to obtain a complete description of the mechanical properties of ScPtSb and YPtSb, we estimated Debye temperature $\theta_{\mathrm{D}}$, which is a fundamental parameter closely related to many physical properties of solids, such as elastic constants, specific heat, melting temperature, from the isotropic longitudinal, transverse and average sound wave velocities $\left(V_{l}, V_{t}\right.$ and $\left.V_{m}\right)$ through the following equa- 
tions [40, 42, 43]:

$$
\left\{\begin{array}{l}
\theta_{\mathrm{D}}=\frac{h}{k_{\mathrm{B}}} V_{m}\left(\frac{3 n}{4 \pi} \frac{N_{\mathrm{A}} \rho}{M}\right)^{1 / 3} \\
V_{m}=\left[\frac{1}{3}\left(2 V_{t}^{-3}+V_{l}^{-3}\right)\right]^{-1 / 3} \\
V_{l}=[(3 B+4 G) / 3 \rho]^{1 / 2} \\
V_{t}=(G / \rho)^{1 / 2}
\end{array}\right.
$$

where, $h$ is Planck constant, $k_{\mathrm{B}}$ is Boltzmann constant, $N_{\mathrm{A}}$ is Avogadro number, $\rho$ is the mass density, $M$ is the molecular weight and $n$ is the number of atoms in the molecule, $B$ is the bulk modulus and $G$ is the shear modulus. The calculated values for the sound velocities $\left(V_{l}, V_{t}\right.$ and $\left.V_{m}\right)$ and Debye temperature $\theta_{\mathrm{D}}$ at zero pressure are reported in table 3 .

Table 3. Calculated longitudinal, transverse and average sound velocities $\left(V_{l}, V_{t}\right.$ and $V_{m}$, in $\left.\mathrm{m} / \mathrm{s}\right)$ and mass density $\rho\left(\mathrm{g} / \mathrm{cm}^{3}\right)$; for the ScPtSb and YPtSb materials.

\begin{tabular}{|c|c|c|c|c|c|}
\hline System & $\rho$ & $V_{l}$ & $V_{t}$ & $V_{m}$ & $\theta_{\mathrm{D}}$ \\
\hline \hline ScPtSb & 9.45 & 4645.31 & 2514.90 & 2806.50 & 301.92 \\
\hline YPtSb & 9.24 & 4422.47 & 2399.87 & 2677.67 & 275.13 \\
\hline
\end{tabular}

The calculated values of Debye temperature $\theta_{\mathrm{D}}$ and the isotropic sound velocities $\left(V_{l}, V_{t}\right.$ and $\left.V_{m}\right)$ of $\mathrm{ScPtSb}$ and $\mathrm{YPtSb}$ at different pressures are shown in figure 8 . The values of the aforementioned parameters increase with an increasing pressure for the ScPtSb and YPtSb compounds. The calculated Debye temperature at zero pressure for $\mathrm{ScPtSb}$ is equal to $301.92 \mathrm{~K}$, which is in acceptable agreement with a reported theoretical value of $311.44 \mathrm{~K}$ [15]. Calculated variation of Debye temperature with pressure is a prediction and there are no corresponding experimental or theoretical results in the literature. The Debye temperature and the sound velocity of ScPtSb at $0 \mathrm{~K}$ and $0 \mathrm{GPa}$ are larger than those of YPtSb. Thus, one can conclude that the propagation velocity sound in $\mathrm{ScPtSb}$ is larger than that in YPtSb. Pressure dependences of $\theta_{\mathrm{D}}$ and sound velocity are well adjusted by a second order polynomial for ScPtSb and YPtSb as follows:

$$
\left\{\begin{array} { l } 
{ \theta _ { \mathrm { D } } = 3 0 1 . 9 2 + 2 . 4 8 P - 0 . 0 3 4 P ^ { 2 } , } \\
{ V _ { l } = 4 6 4 5 . 3 1 + 6 6 . 4 3 P - 0 . 6 5 P ^ { 2 } , } \\
{ V _ { t } = 2 5 1 4 . 9 0 + 1 9 . 5 6 P - 0 . 2 7 P ^ { 2 } , } \\
{ V _ { m } = 2 8 0 6 . 5 0 + 2 3 . 1 4 P - 0 . 3 1 P ^ { 2 } , }
\end{array} \quad \left\{\begin{array}{l}
\theta_{\mathrm{D}}=275.13+3.26 P-0.041 P^{2}, \\
V_{l}=4422.47+75.83 P-0.79 P^{2} \\
V_{t}=2399.87+27.49 P-0.34 P^{2} \\
V_{m}=2677.67+31.87 P-0.39 P^{2}
\end{array}\right.\right.
$$

It is important to evaluate another parameter, namely the elastic anisotropy in materials, because it has an important implication in the engineering science as well as on the nanoscale precursor textures in alloys [44, 45], the elastic anisotropy has an important influence on the induction of microcracks in materials [40, 46]. In a cubic crystal, Zener's $\left(A_{Z}\right)$ [47] and Every's $\left(A_{E}\right)$ [48] elastic anisotropy factors are defined as follows:

$$
\left\{\begin{array}{l}
A_{Z}=2 C_{44} /\left(C_{11}-C_{12}\right) \\
A_{E}=\left(C_{11}-C_{12}-2 C_{44}\right) /\left(C_{11}-C_{44}\right) .
\end{array}\right.
$$

For a completely isotropic material, $A_{E}=0$ and $A_{Z}=1$, and thus, the degree of the deviation of $A_{E}$ from zero and $A_{Z}$ from unity measures the degree of elastic anisotropy. The calculated Zener anisotropy factor is equal to 1.43 for $\mathrm{ScPtSb}$ and 1.20 for $\mathrm{YPtSb}$ and the Every anisotropy factor is equal to -0.34 for $\mathrm{ScPtSb}$ and 0.25 for $\mathrm{YPtSb}$, indicating that both studied compounds are characterized by a certain degree of elastic anisotropy. 

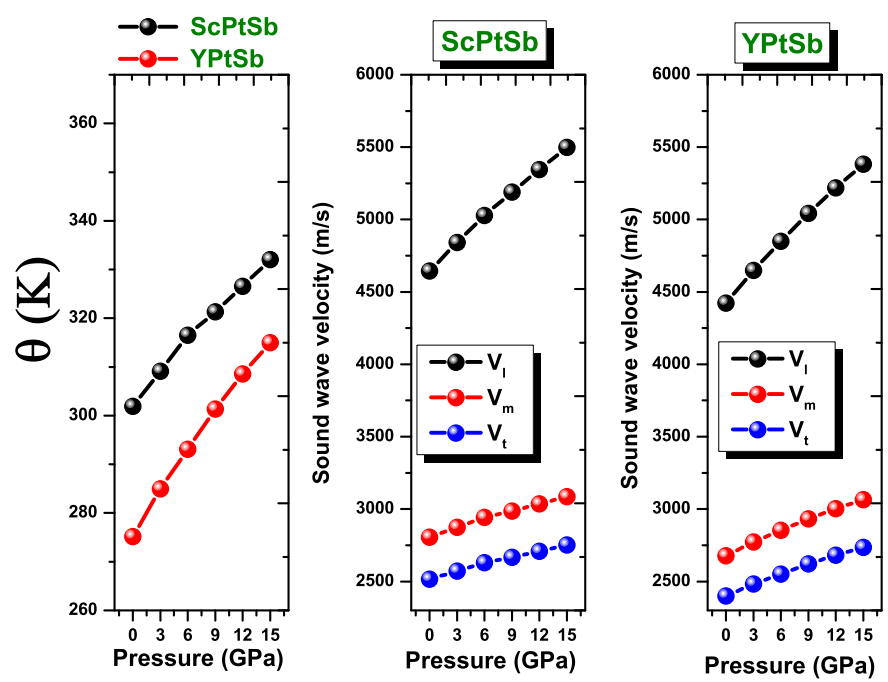

Figure 8. (Colour online) Calculated pressure dependence of Debye temperature $\theta_{\mathrm{D}}$ and the isotropic sound velocity (longitudinal $V_{l}$, transverse $V_{t}$ and average $V_{m}$ ) for the cubic compounds $\mathrm{ScPtSb}$ and YPtSb.

\subsection{Electronic properties}

In order to make sense of the electronic behavior of the ScPtSb and YPtSb half-Heusler compounds, we calculated their band structures along the high symmetry directions, $(W-L-\Gamma-X-W-K)$ in the Brillouin zone using the GGA-PBEsol approach (see figure 9). As can be seen from figure 9, the valence band maximum occurs in the $\Gamma$-point for both compounds, while the conduction band minimum occurs at the $\mathrm{X}$-point for $\mathrm{ScPtSb}$ and at $\Gamma$-point for YPtSb. Thus, $\mathrm{ScPtSb}$ is an indirect band gap semiconductor of $\Gamma-X$ type, while YPtSb is a direct band gap semiconductor of $\Gamma-\Gamma$ type. The calculated fundamental band gap is equal to $0.55 \mathrm{eV}$ for $\mathrm{ScPtSb}$ and $0.136 \mathrm{eV}$ for YPtSb. Our calculated band gap for ScPtSb $(0.55 \mathrm{eV})$ is somewhat larger than that of previous calculations $(0.49 \mathrm{eV})$ [49]. This discrepancy can be attributed to the difference between calculation settings.

Figure 10 shows the total and partial electron density of states (DOS) of ScPtSb and YPtSb at zero and $15 \mathrm{GPa}$. The general profiles of both compounds are almost similar with some differences in band splitting and peaks position. The conduction bands of the ScPtSb (YPtSb) compound are dominantly originated from the Sc-3d (Y-4d) with small contribution from Sb-5p. The valence bands located between 0 and $12 \mathrm{eV}$ are composed of a mixture of $\mathrm{Sc} / \mathrm{Y}, \mathrm{Pt}$ an Sb electronic states. One notes an hybridization between the Pt-5d and Sb-5p, indicating the covalent nature of the Pt-Sb bond. A sharp peak located around $-10.5 \mathrm{eV}$ and $-10 \mathrm{eV}$ originates from the $\mathrm{Sb}-5 s$ state. As shown in figure 10 (TDOS), the bonding peaks near the Fermi level gradually reduce the increasing pressure. The band gaps of both studied compounds increase with an increasing pressure. One can see that the shape of TDOS curves presents a slight change at $15 \mathrm{GPa}$, which means that half-Heusler compounds do not undergo a structural phase transformation under pressure up to $15 \mathrm{GPa}$, and remain structurally stable.

\subsection{Optical properties}

The optical properties of a solid material are defined by the complex dielectric function $\varepsilon(\omega)$ based on the electronic structure and determined by the transitions between the valence bands and the conduction bands. In order to understand the interaction of photons with electrons [50] (interaction of radiation with matter), we calculated the complex dielectric function $\varepsilon(\omega)=\varepsilon_{1}(\omega)+\mathrm{i} \varepsilon_{2}(\omega)$ where the imaginary part of the dielectric function $\varepsilon_{2}(\omega)$ describes the optical absorption in the crystal and $\varepsilon_{1}(\omega)$ the real part describes the dispersive part. The real and imaginary parts of the dielectric function at zero and $15 \mathrm{GPa}$ 

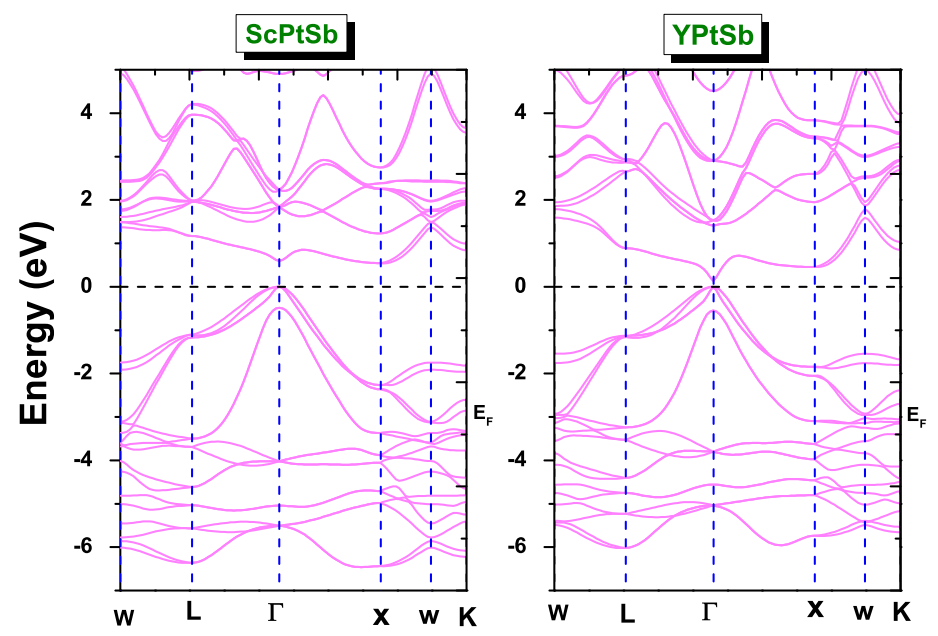

Figure 9. (Colour online) Electronic band dispersion curves along the high symmetry directions in the Brillouin zone for the ScPtSb and YPtSb materials.
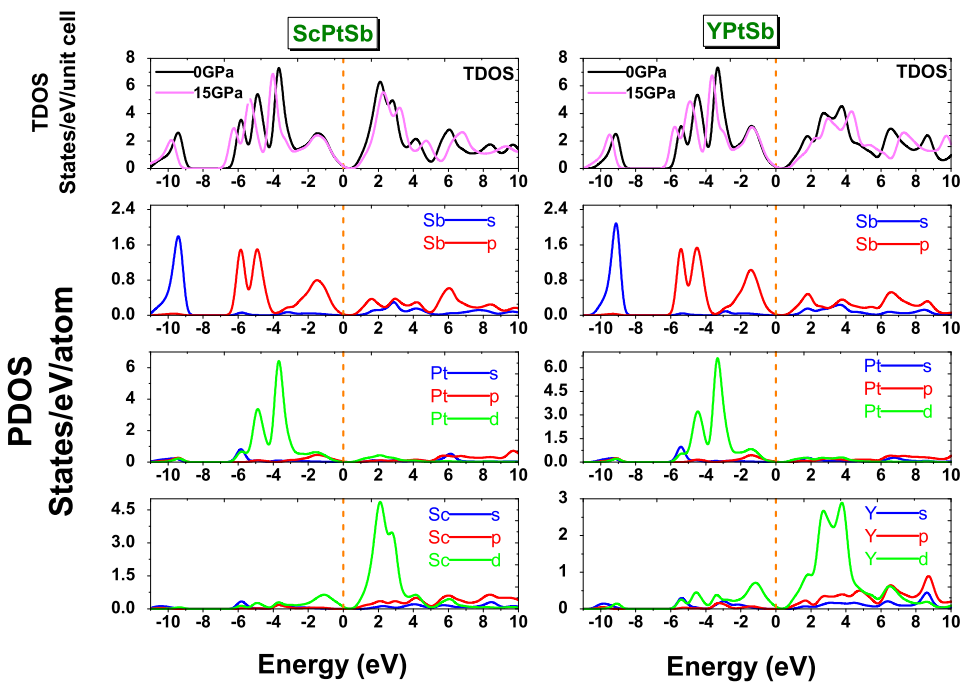

Figure 10. (Colour online) Total (TDOS) and partial (PDOS) densities of states diagrams for the ScPtSb and YPtSb materials.

for $\mathrm{ScPtSb}$ and $\mathrm{YPtSb}$ are shown in figure 11 in an energy range from 0 to $15 \mathrm{eV}$. The zero frequency limit $\varepsilon(0)$ presents an important quantity of $\varepsilon(\omega)$, which depends on the band gap. The calculated value of real part of the dielectric function at the zero-frequency limit $\varepsilon_{1}(0)$ is equal to $14.70(19.88)$ at zero pressure and 13.14 (15.63) at $15 \mathrm{GPa}$ for $\mathrm{ScPtSb}$ (YPtSb). One notes that the $\varepsilon_{1}(0)$ value of $\mathrm{YPtSb}$, which has a band gap of $0.13 \mathrm{eV}$, is lager than that of $\mathrm{ScPtSb}$, which has a band gap of $0.55 \mathrm{eV}$, indicating that the static dielectric function value $\varepsilon(\omega)$ is inversely proportional to the band gap value. This trend is consistent with Penn model [51, 52]: $\varepsilon_{1}(0)=\left(h \omega_{p} / E g\right)^{2}$. The main peak of $\varepsilon_{2}(\omega)$ spectrum occurs at $1.78 \mathrm{eV}(1.23 \mathrm{eV})$ at zero pressure and $2.19 \mathrm{eV}(1.86 \mathrm{eV})$ at $15 \mathrm{GPa}$, for ScPtSb (YPtSb). It is noted that the positions of peaks shift without any important shape change with an increasing pressure from 0 to $15 \mathrm{GPa}$. It is seen that the general profiles of the $\varepsilon_{2}(\omega)$ spectra of the studied compounds are similar. 
The highest peak in the spectrum of the imaginary part of the dielectric function $\varepsilon_{2}(\omega)$ (first critical point) for $\mathrm{ScPtSb}(\mathrm{YPtSb})$ is at $2.71 \mathrm{eV}(2.48 \mathrm{eV})$ at zero pressure and $3.12 \mathrm{eV}(2.84 \mathrm{eV})$ at $15 \mathrm{GPa}$. The critical point known as the fundamental absorption edge [53], which gives the threshold for direct optical interband transition between the topmost valence ( $p$ states of Sb atom) and the lowest conduction band ( $d$ states of $\mathrm{Sc} / \mathrm{Y}$ atoms) is located at around $0.2 \mathrm{eV}(0.1 \mathrm{eV})$ at $0 \mathrm{GPa}$ pressure and $1.06 \mathrm{eV}(0.53 \mathrm{eV})$ at $15 \mathrm{GPa}$ pressure for $\mathrm{ScPtSb}$ and $\mathrm{YPtSb}$, respectively.
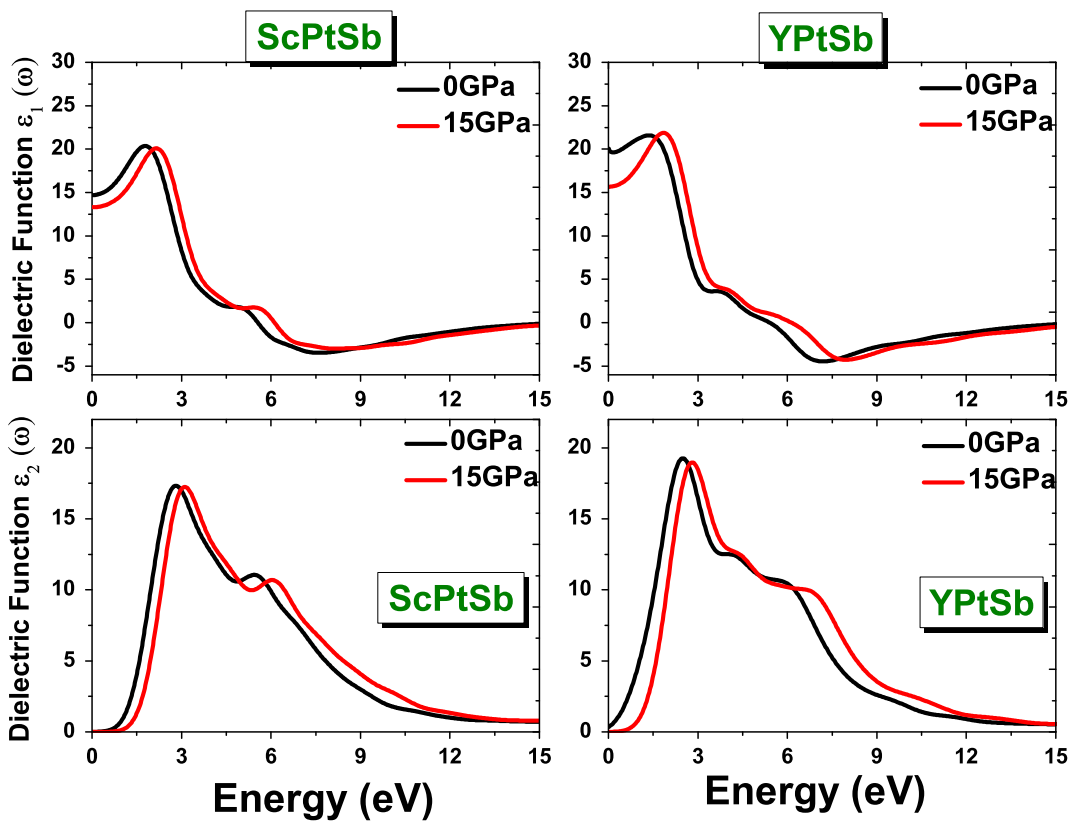

Figure 11. (Colour online) Calculated imaginary part $\varepsilon_{2}(\omega)$ and real part $\varepsilon_{1}(\omega)$ for the ScPtSb and YPtSb materials.

All-important linear optical constants can be derived from the real and imaginary parts of the dielectric function $\varepsilon(\omega)$, namely the electron energy loss function $L(\omega)$, the refractive index $n(\omega)$, extinction coefficient $k(\omega)$, optical reflectivity $R(\omega)$ and absorption coefficient $\alpha(\omega)$ through the following relations [54, 55]:

$$
\left\{\begin{array}{l}
n(\omega)=\frac{1}{\sqrt{2}}\left[\sqrt{\varepsilon_{1}^{2}(\omega)+\varepsilon_{2}^{2}(\omega)}+\varepsilon_{1}(\omega)\right]^{1 / 2}, \\
k(\omega)=\frac{1}{\sqrt{2}}\left[\sqrt{\varepsilon_{1}^{2}(\omega)+\varepsilon_{2}^{2}(\omega)}-\varepsilon_{1}(\omega)\right]^{1 / 2}, \\
R(\omega)=\left|\left[\varepsilon(\omega)^{1 / 2}-1\right] /\left[\varepsilon(\omega)^{1 / 2}+1\right]\right|^{2}, \\
L(\omega)=\varepsilon_{2}(\omega) /\left[\varepsilon_{1}^{2}(\omega)+\varepsilon_{2}^{2}(\omega)\right], \\
\alpha(\omega)=\sqrt{2} \omega\left[\sqrt{\varepsilon_{1}^{2}(\omega)+\varepsilon_{2}^{2}(\omega)}-\varepsilon_{1}(\omega)\right]^{1 / 2} .
\end{array}\right.
$$

Figures 12 and 13 show the calculated spectra of the absorption coefficient, refractive index, extinction coefficient, reflectivity and loss-energy function in an energy range from 0 to $15 \mathrm{eV}$ at zero pressure and $15 \mathrm{GPa}$. To the best of our knowledge, there are no available theoretical or experimental optical spectra in the literature for YPtSb.

As can be seen from figure 12 , the value of refractive index at zero pressure $n(0)$ for $\mathrm{ScPtSb}(\mathrm{YPtSb})$ is equal to $3.84(4.43)$ at zero pressure and $3.60(3.93)$ at $15 \mathrm{GPa}$, indicating that the static refractive index 


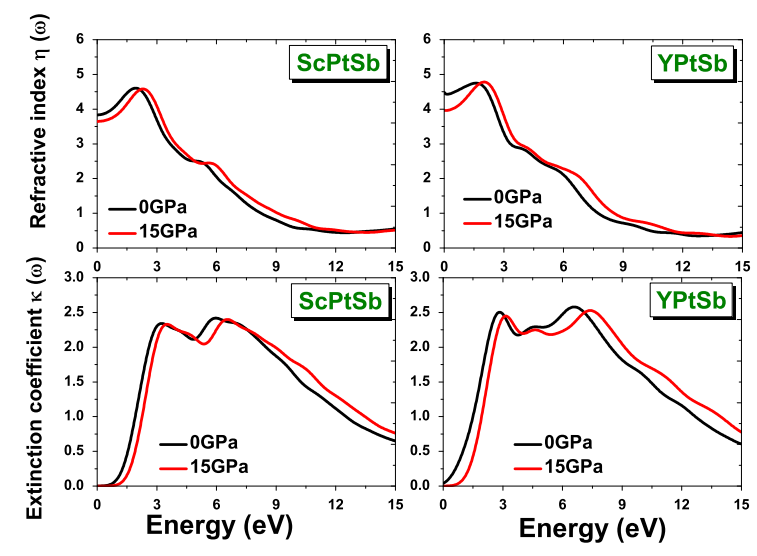

Figure 12. (Colour online) Calculated refractive index $\eta(\omega)$ and extinction coefficient $\kappa(\omega)$ for the $\mathrm{ScPtSb}$ and YPtSb materials.

$n(0)$ decreases with an increasing pressure. The refractive index $n(\omega)$ decreases to attain a minimum level of $0.33(0.26)$ at $11.74(12.37) \mathrm{eV}$ at zero pressure and at $12.62(15) \mathrm{eV}$ at $15 \mathrm{GPa}$ for ScPtSb (YPtSb). The extinction coefficient $k(\omega)$ reaches a maximum of $2.31(2.50)$ at approximately $3.12 \mathrm{eV}(2.81 \mathrm{eV})$ at zero pressure and $2.32(2.43)$ at approximately $3.48 \mathrm{eV}(3.11 \mathrm{eV})$ at $15 \mathrm{GPa}$ pressure for ScPtSb (YPtSb).

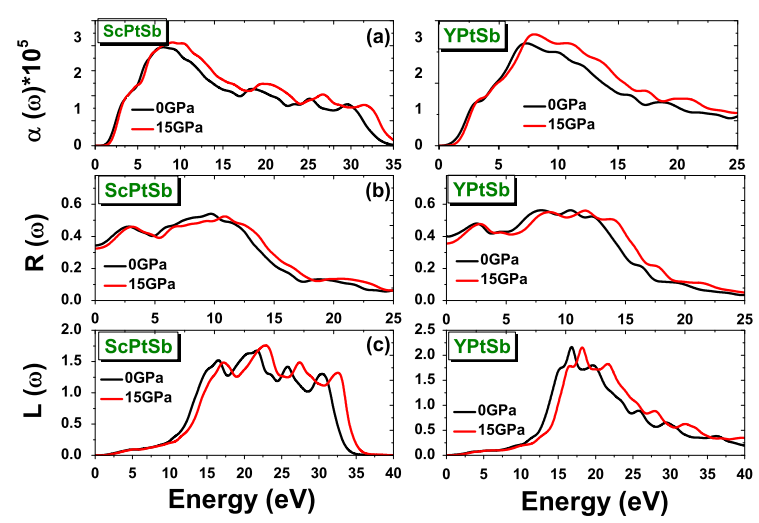

Figure 13. (Colour online) Calculated optical constants for the ScPtSb and YPtSb materials: (a) absorption, (b) reflectivity and (c) energy-loss spectrum.

The absorption coefficient is an important optical parameter which provides useful information on the relative absorption of the radiation energy per length unit inside a medium [56]. Figure 13 (a) shows that the absorption spectrum starts to increase when the photon energy is higher than the absorption edge, which is the typical characteristic of semiconductors and insulators compounds. The absorption spectrum increases rapidly with the wide absorption spectrum of ScPtSb (YPtSb) compounds located about $0-35 \mathrm{eV}$ at zero pressure and about $0-36 \mathrm{eV}$ at $15 \mathrm{GPa}$ pressure, showing the interband transitions from occupied states in valence bands to unoccupied states in conduction bands. These compounds are not a transparent crystal in the interval $0-35 \mathrm{eV}$ but present a noticeable absorption in the visible and far-ultraviolet range, so where the reflectivity and absorption of these compounds become very small (above $36 \mathrm{eV}$ ), these compounds become transparent. The maximum absorption of ScPtSb (YPtSb) occurs in the interval of 7.22-8.88 eV (6.46-8.01 eV) at zero pressure and 7.78-10.95 eV (7.2-8.96 eV) at 
15 GPa pressure. The reflectivity spectrum depends mainly on the incident photon energy. The calculated reflectivity of this $R(\omega)$ spectrum of ScPtSb (YPtSb) is shown in figure 13 (b). The static reflectivity at zero frequency of ScPtSb compound is less than the corresponding value of $\mathrm{YPtSb}$, the maximum reflectivity value of about $0.54(0.56)$ for photon energies $9.71 \mathrm{eV}(7.86 \mathrm{eV})$ at zero pressure and about $0.52(0.56)$ for photon energies $11.08 \mathrm{eV}(11.56 \mathrm{eV})$ at $15 \mathrm{GPa}$ pressure for the both compounds ScPtSb (YPtSb), respectivily. The reflectivity spectra decrease abruptly of these compounds after these energy ranges and reach zero above $35 \mathrm{eV}$, due to the excitations of collective plasma resonance. To further study, we have investigated the $L(\omega)$ loss energy function, is a significant fundamental factor to explain the energy loss of a fast electron traversing in a material, such as interactions including the interband and intraband transitions, phonon excitations, plasmon excitations [55]. The energy loss function, $L(\omega)$, of $\mathrm{ScPtSb}$ (YPtSb) compounds is calculated and shown in figure 13 (c). This function has the main peak which takes place in plasma frequency found to be at $21.14 \mathrm{eV}(16.83 \mathrm{eV})$ at zero pressure and $22.92 \mathrm{eV}(18.21 \mathrm{eV})$ at $15 \mathrm{GPa}$ pressure for $\mathrm{ScXSb}$ (YPtSb), respectively, which corresponds to the abrupt reduction of reflectivity, and the plasma frequency increases going from ScPtSb to YPtSb.

\section{Conclusions}

In summary, we report a comprehensive theoretical investigation of the structural, elastic, electronic and optical properties of the half-Heusler compounds ScPtSb and YPtSb using the pseudopotential plane wave in the framework of density functional theory with the GGA-PBEsol functional. (i) The calculated equilibrium lattice parameters are in good agreement with the available experimental and theoretical data. (ii) Calculated band structures reveal that ScPtSb is an indirect band gap of $\Gamma-X$ type, while $\mathrm{YPtSb}$ is a direct band gap of $\Gamma-\Gamma$ type. (iii) The band gaps decrease with an increasing pressure for both studied compounds. (iv) The nature of the electronic states in the valence and conduction bands was identified through the calculation the diagrams of the TDOS and PDOS. (v) The elastic constants of both single-crystal and polycrystalline phases of the half-Heusler XPtSb $(\mathrm{X}=\mathrm{Sc}, \mathrm{Y})$ were estimated at zero-pressure and under pressure effect up to $15 \mathrm{GPa}$. It is found that the elastic constants satisfy the mechanical stability criteria in all the considered pressure range. (vi) The Young's modulus, shear modulus, Poisson's ratio, anisotropy factor, sound velocities, and Debye temperature were estimated and they are in acceptable agreement with the available data. It is also found that ScPtSb and YPtSb exhibit a noticeable elastic anisotropy. (vii) According to the empirical rule of Pugh, ScPtSb and YPtSb are ductile materials. (viii) The calculated optical properties, namely the dielectric function, absorption coefficient spectrum, refractive index, extinction coefficient, reflectivity and energy-loss function were obtained for both compounds. It is found that the static dielectric function at zero pressure increases when Sc atom in $\mathrm{ScPtSb}$ is replaced by $\mathrm{Y}$ atom. The dielectric functions increase with an increasing pressure due to the decrease of the optical conductivity and the increase of the band gap. Thus, around zero frequency the optical conductivity of ScPtSb is smaller than that of $\mathrm{YPtSb}$.

\section{Acknowledgements}

This work is supported by PTEA Laboratory, University of Yahia Fares Medea.

\section{References}

1. Graf T., Felser C., Parkin S., Prog. Solid State Chem., 2011, 39, 1-50, doi $10.1016 /$ j.progsolidstchem.2011.02.001.

2. Gofryk K., Kaczorowski D., Plackowski T., Mucha J., Leithe-Jasper A., Schnelle W., Grin Yu., Phys. Rev. B, 2007, 75, 224426, doi $10.1103 /$ PhysRevB.75.224426

3. Hermet P., Niedziolka K., Jund P., RSC Adv., 2013, 3, 22176-22184, doi 10.1039/C3RA43990A

4. Uher C., Yang J., Hu S., Morelli D. T., Meisner G. P., Phys. Rev. B, 1999, 59, 8615, doi $10.1103 /$ PhysRevB.59.8615. 
5. Hohl H., Ramirez A. P., Goldmann C., Ernst G., Wölfing B., Bucher E., J. Phys.: Condens. Matter, 1999, 11, 1697, doi $10.1088 / 0953-8984 / 11 / 7 / 004$

6. Larson P., Mahanti S. D., Sportouch S., Kanatzidis M. G., Phys. Rev. B, 1999, 59, 15660, doi $10.1103 /$ PhysRevB.59.15660

7. Pierre J., Skolozdra R. V., Tobola J., Kaprzyk S., Hordequin C., Kouacou M. A., Karla I., Currat R., LelievreBerna E., J. Alloys Compd., 1997, 262-263, 101, doi 10.1016/S0925-8388(97)00337-X

8. Kandpal H. C., Felser C., Seshadri R., J. Phys. D: Appl. Phys., 2006, 39, 776, doi:10.1088/0022-3727/39/5/S02

9. Larson P., Mahanti S. D., Kanatzidis M. G., Phys. Rev. B, 2000, 62, 12754, doi 10.1103/PhysRevB.62.12754.

10. Tobola J., Pierre J., Kaprzyk S., Skolozdra R. V., Kouacou M. A., J. Magn. Magn. Mater., 1996, 159, 192, doi 10.1016/0304-8853(95)00620-6.

11. Tobola J., Pierre J., J. Alloys Compd., 2000, 296, 243-252, doi:10.1016/S0925-8388(99)00549-6

12. Nowak B., Kaczorowski D., Intermetallics, 2013, 40, 28-35, doi:10.1016/j.intermet.2013.04.001.

13. Singh S., Kumar R., J. Alloys Compd., 2017, 722, 544-548, doi 10.1016/j.jallcom.2017.06.131.

14. Kocak B., Ciftci Y. O., Comput. Condens. Matter, 2018, 14, 176-185, doi 10.1016/j.cocom.2018.01.009

15. Narimani M., Nourbakhsh Z., J. Phys. Chem. Solids, 2017, 102, 121-129, doi $10.1016 /$ j.jpcs.2016.11.014

16. Oestreich J., Probst U., Richardt F., Bucher E., J. Phys.: Condens. Matter, 2003, 15, 635, doi:10.1088/0953$8984 / 15 / 4 / 304$

17. Clark S. J., Segall M. D., Pickard C. J., Hasnip P. J., Probert M. J., Refson K., Payne M. C., Z. Kristallogr. Cryst. Mater., 2005, 220, 567, doi:10.1524/zkri.220.5.567.65075.

18. Perdew J. P., Ruzsinszky A., Csonka G. I., Vydrov O. A., Scuseria G. E., Constantin L. A., Zhou X., Burke K., Phys. Rev. Lett., 2008, 100, 136406, doi:10.1103/PhysRevLett.100.136406.

19. Vanderbilt D., Phys. Rev. B, 1990, 41, 7892, doi $10.1103 /$ PhysRevB.41.7892

20. Monkhorst H. J., Pack J. D., Phys. Rev. B, 1976, 13, 5188, doi 10.1103/PhysRevB.13.5188.

21. Fischer T. H., Almlof J., J. Phys. Chem., 1992, 96, 9768, doi:10.1021/j100203a036

22. Birch F., Phys. Rev., 1947, 71, 809-816, doi 10.1103/PhysRev.71.809

23. Birch F., J. Geophys. Res., 1978, 83, 1257-1268, doi:10.1029/JB083iB03p01257

24. Ambrosch-Draxl C., Sofo J. O., Comput. Phys. Commun., 2006, 175, 1-14, doi:10.1016/j.cpc.2006.03.005

25. Vinet P., Rose J. H., Ferrante J., Smith J. R., J. Phys.: Condens. Matter, 1989, 1, 1941, doi:10.1088/0953$8984 / 1 / 11 / 002$

26. Fu C. L., Ho K. M., Phys. Rev. B, 1983, 28, 5480, doi $10.1103 /$ PhysRevB.28.5480

27. Murnaghan F. D., Proc. Natl. Acad. Sci. U.S.A., 1944, 30, 244-247, doi:10.1073/pnas.30.9.244

28. Harmening T., Eckert H., Pöttgen R., Solid State Sci., 2009, 11, 900, doi $10.1016 /$ j.solidstatesciences.2008.12.007

29. Yang B. J., Li H., Wu T., Zhang W., Chen L., Yang J., Adv. Funct. Mater., 2008, 18, 2880-2888, doi $10.1002 / \mathrm{adfm} .200701369$

30. Li C., Zhao Y. F., Chi B. Q., Gong Y. Y., Sun C. Q., Comput. Condens. Matter, 2014, 1, 8-13, doi $10.1016 /$ j.cocom.2014.08.002

31. Li C., Wen Z., Thin Solid Films, 2013, 546, 436-438, doi:10.1016/j.tsf.2013.04.066

32. Wenski G., Mewis A., Z. Kristallogr. - Cryst. Mater., 1986, 176, 125-134, doi 10.1524/zkri.1986.176.12.125

33. Page Y. L., Saxe P., Phys. Rev. B, 2002, 65, 104104, doi 10.1103/PhysRevB.65.104104

34. Westbrook J. H., Fleischer R. L. (Eds.), Intermetallic Compounds, Vol. 3, John Wiley\&Sons Ltd, Chichester, England, 2000.

35. Liu Q. J., Ran Z., Liu F. S., Liu Z. T., J. Alloys Compd., 2015, 631, 192-201, doi 10.1016/j.jallcom.2015.01.085

36. Voigt W., Lehrbuch der Kristallphysik (Textbook of crystal physics), Teubner, Leipzig, 1928.

37. Hill R., Proc. Phys. Soc. London, Sect. A, 1952, 65, 349-354, doi: $10.1088 / 0370-1298 / 65 / 5 / 307$.

38. Wu Z., Zhao E., Xiang H., Hao X., Liu X., Meng J., Phys. Rev. B, 2007, 76, 054115, doi $10.1103 /$ PhysRevB.76.054115.

39. Pugh S. F., London, Edinburgh Dublin Philos. Mag. J. Sci., 1954, 45, 823-843, doi $10.1080 / 14786440808520496$.

40. Ravindran P., Fast L., Korzhavyi P. A., Johansson B., Wills J., Eriksson O., J. Appl. Phys., 1998, 84, 4891, doi $10.1063 / 1.368733$

41. Nye J. F., Physical Properties of Crystals, Oxford University Press, New York, 1985, doi:10.1088/00319112/36/12/027.

42. Anderson O. L., J. Phys. Chem. Solids, 1963, 24, 909-917, doi 10.1016/0022-3697(63)90067-2.

43. Schreiber E., Anderson O. L., Soga N., Elastic Constants and Their Measurement, McGraw-Hill Companies, New York, 1974, doi $10.1115 / 1.3423687$

44. Lloveras P., Castán T., Porta M., Planes A., Saxena A., Phys. Rev. Lett., 2008, 100, 165707, doi $10.1103 /$ PhysRevLett.100.165707 
45. Pan R. K., Ma L., Bian N., Wang M. H., Li P. B., Tang B. Y., Peng L. M., Ding W. J., Phys. Scr., 2013, 87, 015601, doi $10.1088 / 0031-8949 / 87 / 01 / 015601$

46. Chung D. H., Buessem W. R., In: Anisotropy in Single-Crystal Refractory Compounds, Vahldiek F. W., Mersol S. A. (Eds.), Plenum, New York, 1968. doi 10.1007/978-1-4899-5307-0

47. Zener C., Elasticity and Anelasticity of Metals, University of Chicago Press, Chicago, Illinois, 1948.

48. Every A. G., Phys. Rev. B, 1980, 22, 1746-1760, doi 10.1103/PhysRevB.22.1746.

49. Landau L. D., Lifschitz E. M., Fluid Mechanics, Pergamon Press, New York, 1980.

50. Sun J., Wang H. T., Ming N. B, He J., Tian Y., Appl. Phys. Lett., 2004, 84, 4544, doi 10.1063/1.1758781.

51. Ahmad M., Naeemullah, Murtaza G., Khenata R., Omran S. B., Bouhemadoue A., J. Magn. Magn. Mater., 2015, 377, 204-210, doi $10.1016 /$ j.jmmm.2014.10.108

52. Penn D. R., Phys. Rev., 1962, 128, 2093-2097, doi 10.1103/PhysRev.128.2093

53. Khenata R., Bouhemadou A., Sahnoun M., Reshak A. H., Baltache H., Rabah M., Comput. Mater. Sci, 2006, 38, 29-38, doi:10.1016/j.commatsci.2006.01.013

54. Jana D., Sun C. L., Chen L. C., Chen K. H., Prog. Mater Sci., 2013, 58, 565-635, doi $10.1016 /$ j.pmatsci.2013.01.003

55. Wooten F., Optical Properties of Solids, Academic, New York, 1972.

56. Roknuzzaman M., Islam A. K. M. A., Int. Scholarly Res. Not., 2013, 2013, 646042 doi: $10.1155 / 2013 / 646042$.

\title{
Структурні, пружні, електронні та оптичні властивості напів-Гейслерівських сполук ScPtSb i YPtSb при наявності зовнішнього тиску
}

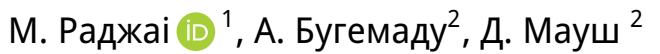 \\ 1 Лабораторія фізики експериментальних технологій та їх застосувань, Університет Медеї, Алжир. \\ 2 Лабораторія розробки та дослідження нових матеріалів, Університет Ферхат Аббас Сетиф 1, Сетиф 19000,
} Алжир.

3 метою дослідження структурних, пружних, електронних та оптичних властивостей напівГейслерівських сполук ScPtSb i YPtSb при наявності зовнішнього тиску, що мають кубічну структуру типу MgAgAs, проведено першопринципні розрахунки з використанням псевдопотенціалу в базисі плоских хвиль у рамках методу апроксимації узагальнених градієнтів. Обчислення проведено з урахуванням спін-орбітальної взаємодії. Розраховані рівноважні сталі ґратки добре узгоджуються з наявними експериментальними і теоретичними значеннями. Жорсткість та механічна стійкість кристалів обговорюється на основі розрахованих пружних сталих та пов'язаних з ними характеристик, таких як модуль об'ємної пружності, модуль зсуву, температура Дебая, коефіцієнт Пуассона, модуль Юнга та ізотропні швидкості звуку. 3 розрахованих електронних зонних структур випливає, що в ScPtSb $\epsilon$ непряма заборонена зона $\Gamma-X$ типу, в той час як у YPtSb присутня пряма заборонена зона типу $\Gamma-\Gamma$. Для обидвох сполук ScPtSb та YPtSb досліджено вплив тиску на оптичні властивості, а саме на діелектричну функцію, спектр поглинання, показник заломлення, коефіцієнт екстинкції, коефіцієнт відбиття та спектр енергетичних втрат.

Ключові слова: ScPtSb, YPtSb, метод PP-PW, оптичні властивості, електронні властивості, модулі пружності, першопринципні розрахунки 